\title{
DESIGN OF A PIPING INSPECTION ROBOT BY OPTIMIZATION APPROACH
}

\author{
Swaminath Venkateswaran ${ }^{1,2}$,Damien Chablat ${ }^{2,3 *}$, Pol Hamon ${ }^{2}$ \\ ${ }^{1}$ Ecole Centrale de Nantes, Nantes, 44321 France \\ ${ }^{2}$ Laboratoire des Sciences du Numérique de Nantes (LS2N), UMR CNRS 6004, Nantes, 44300, France \\ ${ }^{3}$ Centre National de la Recherche Scientifique (CNRS), Nantes, 44321, France \\ Emails: \{swaminath.venkateswaran, damien.chablat, pol.hamon\}@ls2n.fr
}

\begin{abstract}
This article presents an optimization approach for the design of an inspection robot that can move inside variable diameter pipelines having bends and junctions. The inspection robot uses a mechanical design that mimics the locomotion of a caterpillar. The existing prototype developed at LS2N, France is a rigid model that makes it feasible for working only inside straight pipelines. By the addition of a tensegrity mechanism between motor units, the robot is made reconfigurable. However, the motor units used in the prototype are oversized to pass through pipe bends or junctions. An optimization approach is employed to determine the dimensions of motors and their associated leg mechanisms that can overcome such bends. Two optimization problems are defined and solved in this article. The first problem deals with the determination of motor sizing without leg mechanisms. The second problem deals with the determination of sizing of the leg mechanism with respect to the dimensions of motor units obtained from the first problem. A $3 D$ model of the optimized robot design is then realized using CAD software.
\end{abstract}

\section{INTRODUCTION}

Manual intervention of pipelines in industries such as nuclear or chemical is cumbersome and risky as it may lead to loss of human life or causes long term radiation effects. Inspection robots play a vital role in such situations as they not only reduce human effort but also can perform a given task with better accuracy. In-pipe inspection robots can be classified [1] viz: Pig type [2], Wheel type [3], Caterpillar type [4], Wall-press type [5], Walking type [6], Inchworm type [7] and Screw type [8]. A bioinspired piping inspection robot was designed and developed at LS2N, France [9]. This robot accomplishes the locomotion of a caterpillar in six-steps to move inside a pipeline. Using a slotfollower leg mechanism, the robot attains static postures to establish contact with pipeline walls. The leg mechanisms are capable of working between 40-94 mm diameter straight pipelines [10]. However, the robot is a rigid model and is thus limited to straight pipelines. Also, the spindle drive used in the prototype allows the robot to move at a very low speed of $0.43 \mathrm{~mm} / \mathrm{s}$ [11]. By the addition of a tensegrity mechanism between the motor modules, the robot is made reconfigurable [12]. The dimensions of the existing motor units are oversized and might restrict the reconfigurable robot to pass through pipe bends at $90^{\circ}$. In this article, an optimization approach is followed to determine the motor sizing that will be capable of overcoming pipe bends. Optimization algorithms can be classified into deterministic and heuristic approaches [13]. The deterministic optimization approach solves problems in a structured manner that leads to efficient solutions with respect to design variables and constraints. On the other hand, heuristic/evolutionary algorithms operate on randomness. Based on the complexity of the design problem, the heuristic approach requires lesser computational times over the deterministic approach [14]. However, there are possibilities that both the algorithms can lead to solutions trapped in a local minimum. For the bio-inspired robot under study, a deterministic approach using fmincon is carried out in MATLAB. The problem is solved for a planar test bench where the robot is constructed as multi-

*Address all correspondence to this author 
body blocks. Two optimization problems have been solved for determining the optimal robot assembly. The first problem deals with the determination of motor sizes of each module without the presence of leg mechanisms. From the results of optimization, the motors and gear units are identified with catalog products of Maxon based on dimensions, velocity and force factors. Also, the first optimization permits to set the diameter range of pipelines for the robot. For studying the area occupied by the leg mechanisms, the second optimization problem is then carried out which helps in determining the geometry of the slot-follower leg mechanism. The area occupied by the leg mechanism is studied by accommodating the mechanism on the motor sizing determined from the results of the first problem. A 3D model of the optimized robot design is realized using CATIA software.

The outline of the article is as follows. In the following section, the architecture of the robot is presented. The next section deals with the overall problem definition of the optimization process, the representation of the test bench and modeling of robot using MATLAB. The subsequent section deals with the first optimization problem methodology and results. Followed by that the second optimization problem is defined and solved. The article then ends with conclusions.

\section{ARCHITECTURE OF THE ROBOT}

The bio-inspired robot developed at LS2N, France comprises three modules that ensure clamping and elongation phases for accomplishing the locomotion of a caterpillar. Each module consists of a DC brushless motor coupled with a spindle drive to convert rotary motion to prismatic movement $[9,10]$. The front and rear modules have 3 sets of slot-follower leg mechanisms each to have contact with the inner walls of pipelines at all instances of locomotion. The central module takes care of the elongation and retraction phases. Through the study of design issues namely passive compliance, active compliance and tilt limits [12], a tensegrity mechanism was proposed to be introduced to the robot to make it reconfigurable. The tensegrity mechanism comprises a passive universal joint and three tensions springs. While passing through pipe bends at $90^{\circ}$, the mechanism operates in a passive mode to overcome the bend. In the event of a junction or T-union inside the pipeline, cables that pass through springs of the mechanism can be actuated through external motors for tilting the tensegrity mechanism along a given direction. By workspace analysis with respect to joint limits of springs, an inverse pendulum configuration is employed for the tensegrity mechanism [15]. A 3D model of the bio-inspired robot with the tensegrity mechanism is represented in Fig. 1. In Fig. 1, $T_{1}$ and $T_{2}$ represent the tensegrity mechanisms. The CAD model depicted in Fig. 1 is constructed using the motors employed in the rigid prototype. If this reconfigurable robot is employed within a pipeline having diameters less than $100 \mathrm{~mm}$, the modules can get trapped in the bends or elbows as the dimensions of the mo-

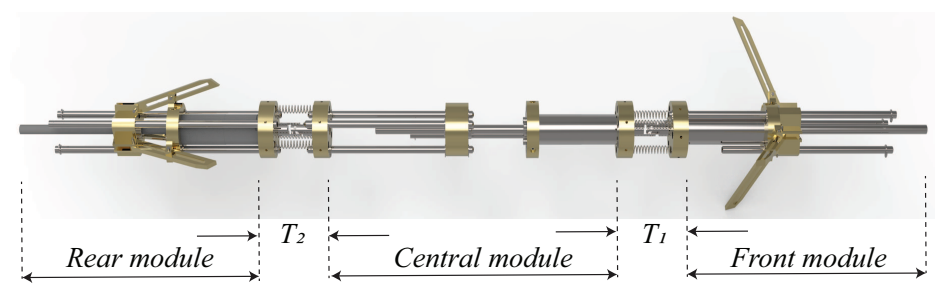

FIGURE 1: 3D model of the existing bio-inspired robot with the tensegrity mechanisms

tor units prove to be oversized. For determining the dimensions of motors and leg mechanisms that can overcome such bends, an optimization approach is being implemented.

\section{PROBLEM DEFINITION}

In this article, two optimization problems are solved to determine the robot assembly. The first problem deals with the determination of motor sizes of each module without the presence of leg mechanisms. To simplify the computation, the dimensions of the tensegrity mechanism are considered fixed. The optimization problem is tested for pipeline diameters ranging from 70 to $160 \mathrm{~mm}$. From the results of optimization, a suitable motorspindle combination unit is identified from the catalog of Maxon. The optimization problem also addresses the following factors for the reconfigurable prototype: i) Velocity- An alternate spindle drive unit with lower gear reduction ratio compared to the existing prototype (455:1) to accomplish faster displacements during locomotion, ii) Hall sensors- A DC motor coupled with hall sensor units which will be useful for control phase of the robot while working inside an unknown environment [16]. Once the motor unit has been identified, the second problem is carried out for the results of optimization of the first problem for determining the size of the slot-follower leg mechanism.

\subsection{Test bench}

The optimization problems are solved in a planar test bench. The test bench consists of a horizontal pipe section, a $90^{\circ}$ elbow and a vertical pipe section. The optimal sizing is determined especially at the bend section wherein collision of modules against the walls of the pipelines is verified. The test bench for the optimization problem is represented in Fig. 2. The test pipe represented in Fig. 2a is generally manufactured by Numerical Control (NC) bending processes using a roller with its origin at $\mathbf{o}$ and flexible mandrels [17]. The coordinate of point $\mathbf{o}$ is given by $[1.5 \mathrm{~d}, 1.5 \mathrm{~d}]$ where $d$ is the diameter of the pipe. With respect to $\mathbf{o}$, the trajectories of the test bench are classified as the inner portion, center line radius (CLR) and the outer portion. The bending radius of these trajectories represented in Fig. $2 \mathrm{~b}$ is given by $\left[r_{1}, r_{2}, r_{3}\right]=[d, 1.5 d, 2 d]$. The optimization problem is solved for 

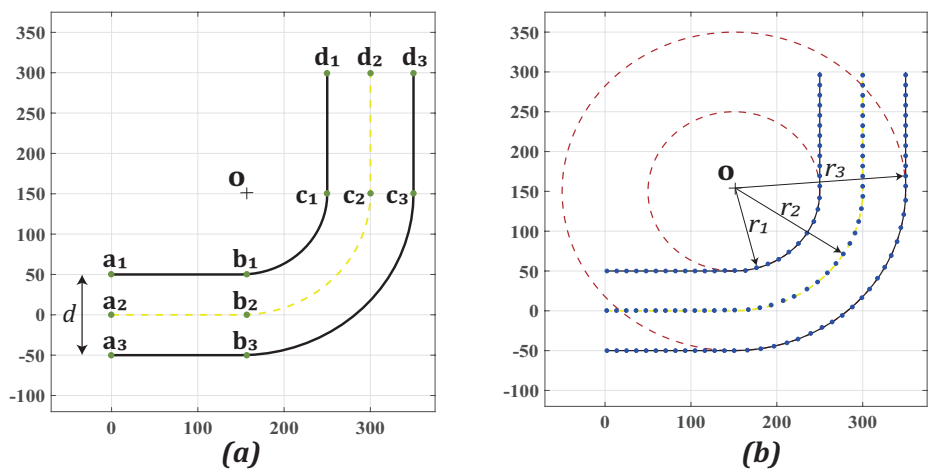

(b)

FIGURE 2: Overview of (a) the test bench for optimization and (b) parametrization of the test bench

a fixed diameter value at any instant. The values considered for $d$ are $[70,80,90,100,110,120,130,140,150,160] \mathrm{mm}$. The pipeline geometry is constructed in MATLAB by sub-dividing into straight and bent cross-sections. The coordinate system for the test bench with respect to o for Fig. $2 \mathrm{a}$ is given in Table 1.

\section{TABLE 1: COORDINATE SYSTEM OF PIPE GEOMETRY}

\begin{tabular}{cccc}
\hline Coordinate & Inner $(\mathrm{t}=1)$ & Center $(\mathrm{t}=2)$ & Outer $(\mathrm{t}=3)$ \\
\hline $\mathbf{a}_{i}$ & $r_{1}[0,0]$ & $r_{2}[0,0]$ & $r_{3}[0,0]$ \\
$\mathbf{b}_{i}$ & $r_{1}[1,0]$ & $r_{2}[1,0]$ & $r_{3}[1,0]$ \\
$\mathbf{c}_{i}$ & $r_{1}[2,1]$ & $r_{2}[2,1]$ & $r_{3}[2,1]$ \\
$\mathbf{d}_{i}$ & $r_{1}[2,2]$ & $r_{2}[2,2]$ & $r_{3}[2,2]$ \\
\hline
\end{tabular}

A discretization is done between each coordinate wherein the continuous trajectory is being subdivided into discrete counterparts. This discretization is being applied to the walls of the pipelines as well as the CLR and they are represented by blue dotted lines in Fig. 2b. The inner and outer portion discretization is employed to check for collision against modules during locomotion. The discretization of the CLR is mainly used to move the robot and also to rotate the modules. The discretization equations for the straight sections are given by

$$
\begin{aligned}
& \mathbf{m}=\mathbf{a}_{t}+\operatorname{disc}\left(\mathbf{b}_{t}-\mathbf{a}_{t}\right) \\
& \mathbf{m}=\mathbf{c}_{t}+\operatorname{disc}\left(\mathbf{d}_{t}-\mathbf{c}_{t}\right)
\end{aligned}
$$

where disc $=0: 0.01: 1$ and $t=1,2,3$

In Eqn. (1) \& Eqn. (2), m indicates the coordinates of dis- cretized points between the start and end points of the straight sections. The bend section of pipe with the discretized points between $\mathbf{b}_{t}-\mathbf{c}_{t}$ is constructed using the equation

$$
\begin{array}{r}
\mathbf{m}_{j}=\left[r_{t} \sin \left(\frac{j \pi}{40}\right),-r_{t} \cos \left(\frac{j \pi}{40}\right)\right] \\
\text { where } j=0 \text { to } 20 \text { and } t=1,2,3
\end{array}
$$

\subsection{Modeling of robot and design variables}

The robot is modeled as multi-body planar blocks with a tensegrity mechanism between each module. Each module comprises of a DC-motor coupled with a spindle drive. The robot geometry considered for optimization is represented in Fig. 3. The reference frame of the robot $\Sigma_{0}$ is fixed at the center of gravity (CG) position of the central module. The movement of the robot is simulated in MATLAB by displacing the CG of the reference frame to each discretized point on the CLR. For any position of the robot on a given discretized point on the CLR, the rotation angle is applied to the other modules as well as to the tensegrity mechanisms to accomplish the rotation while passing through pipe bends. The rotation of modules during locomotion inside the test bench is accomplished as described below in Algorithm 1.

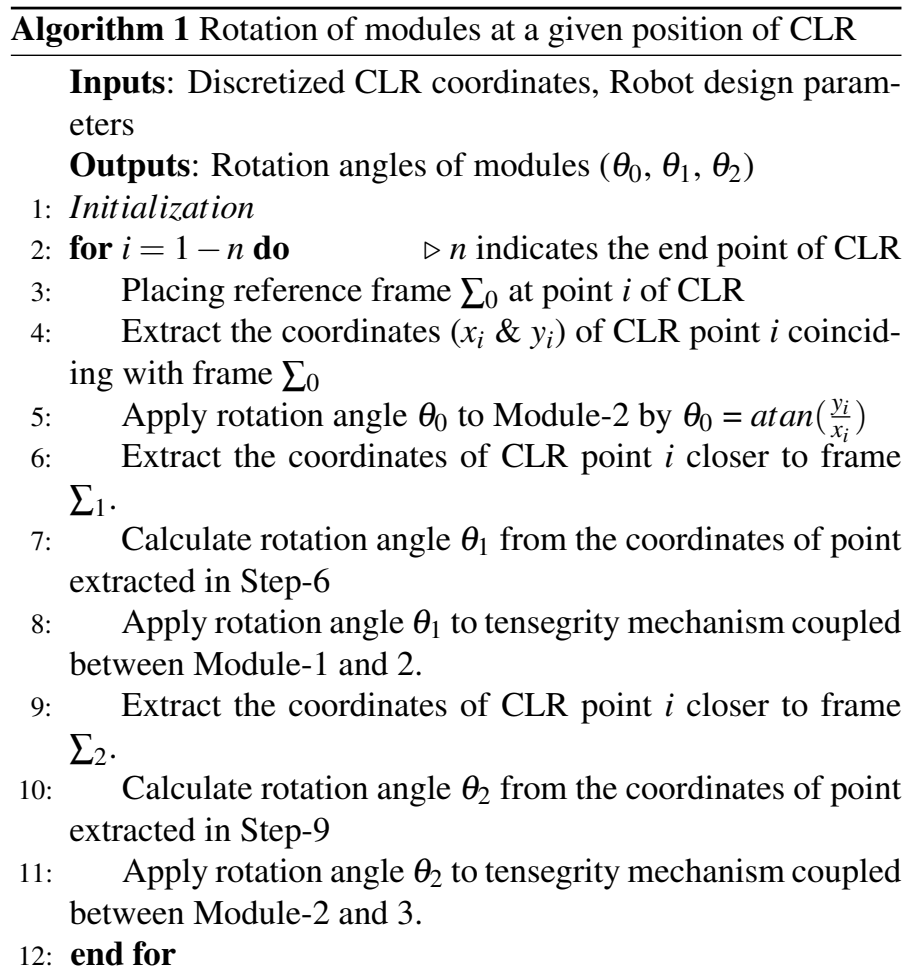




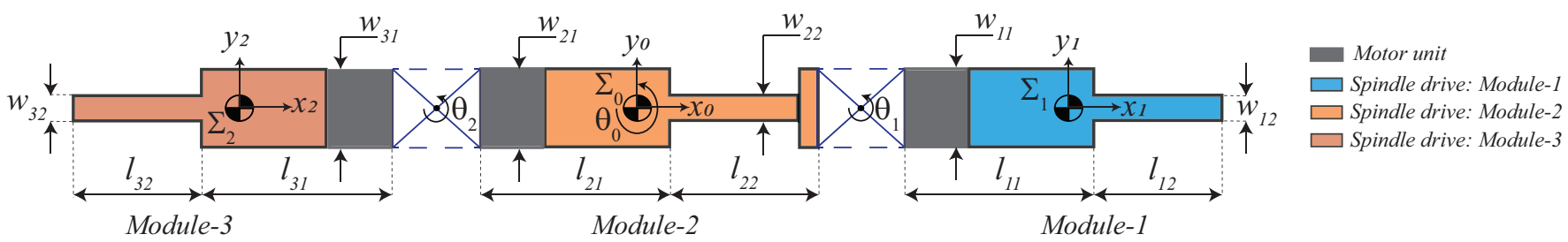

FIGURE 3: Representation of the robot assembly with various design parameters for the optimization problem

The design variables for the motor-gear unit are given by $\left[l_{k 1}, w_{k 1}\right]$. The screw unit of the spindle drive is defined by $\left[l_{k 2}, w_{k 2}\right]$. Here $k$ indicates a module and it assumes values from 1 to 3 . These design parameters are also represented in Fig. 3 for each module. The dimensions of the tensegrity mechanism are fixed and are defined by $\left[2 r_{f}, 2 r_{f}\right]$. An inverse pendulum configuration with a value of $11 \mathrm{~mm}$ for $r_{f}$ is chosen [15].

\section{FIRST OPTIMIZATION PROBLEM: MOTOR SIZING}

A single-objective optimization problem subject to constraints is solved using MATLAB. The leg mechanisms are not taken into account for the first optimization problem and an optimal motor sizing is determined at all discretized positions of CLR by avoiding collisions against pipeline walls. As the central module takes care of elongation and retraction phases, it is assumed for the computation that this module remains in a fully extended phase at all instances of simulation.

\subsection{Objective function}

The objective function of the problem aims at maximizing the area of the motor units. Since the dimensions of the tensegrity mechanism are fixed, their areas are not taken into account. The area of the robot at a given position of CLR is given by

$$
f u n 1=\sum_{k=1}^{3}\left(l_{k 1} w_{k 1}+l_{k 2} w_{k 2}\right)
$$

where $k$ indicates the module

The area of motor units is estimated as per Eqn. (4). The global sum of fun 1 from Eqn. (4) is calculated throughout the discretized points of the CLR and it will be maximized.

\subsection{Constraint function}

For each position of the robot on the CLR, the collision of modules is checked against pipeline walls. Inequality constraints are defined in the optimization problem which will ensure that the modules avoid collision during movement inside the test bench, especially at the bends. A complete discretization of each module is performed. The discretization equation is similar to Eqn. (1) \& Eqn. (2). The discretized robot modules inside the test bench are represented in Fig. 4. For each position of

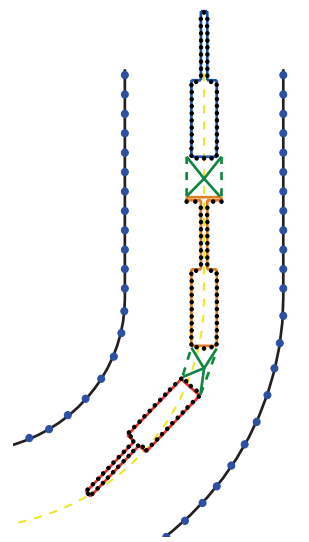

(a)

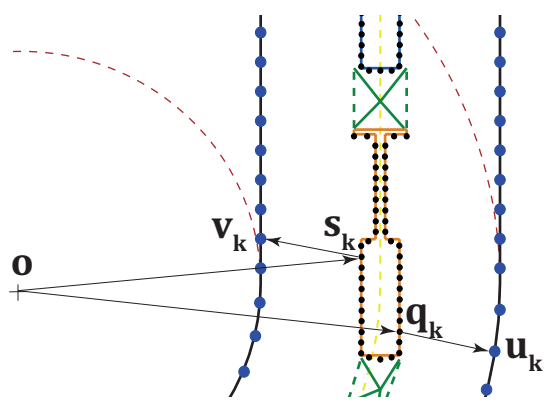

(b)
FIGURE 4: (a) Discretized robot assembly and (b) extraction of coordinates from the discretized model for defining constraints

reference frame $\sum_{0}$ on the discretized CLR trajectory, the points closer and farther on each module from the point $\mathbf{o}$ are extracted. These points can be extracted using the min and max function of MATLAB. The representation of the points closer $\left(\mathbf{s}_{k}\right)$ and farther $\left(\mathbf{q}_{k}\right)$ from point $\mathbf{o}$ on a module is shown in Fig. 4b. Followed by that, the point $\mathbf{v}_{k}$ on the inner portion closer to $\mathbf{s}_{k}$ and the point $\mathbf{u}_{k}$ on the outer portion closer to $\mathbf{q}_{k}$ are extracted using the min function. With the extracted set of points, the inequality constraints are defined in MATLAB which ensures that there exist no collisions between modules and pipeline walls. The constraint equations at a given point on CLR are thus defined by

$$
\begin{array}{r}
g_{k}:\left\|\mathbf{o q}_{k}\right\| \leq\left\|\mathbf{o u}_{k}\right\| \\
g_{k+1}:\left\|\mathbf{o s}_{k}\right\| \geq\left\|\mathbf{o v}_{k}\right\| \\
\text { where } k=1,2,3
\end{array}
$$


Throughout the locomotion sequence, the collision of each module against pipeline walls is checked using Eqn. (5) \& Eqn. (6). The pseudo-code for the constraint function and its working in MATLAB is provided in Algorithm 2.

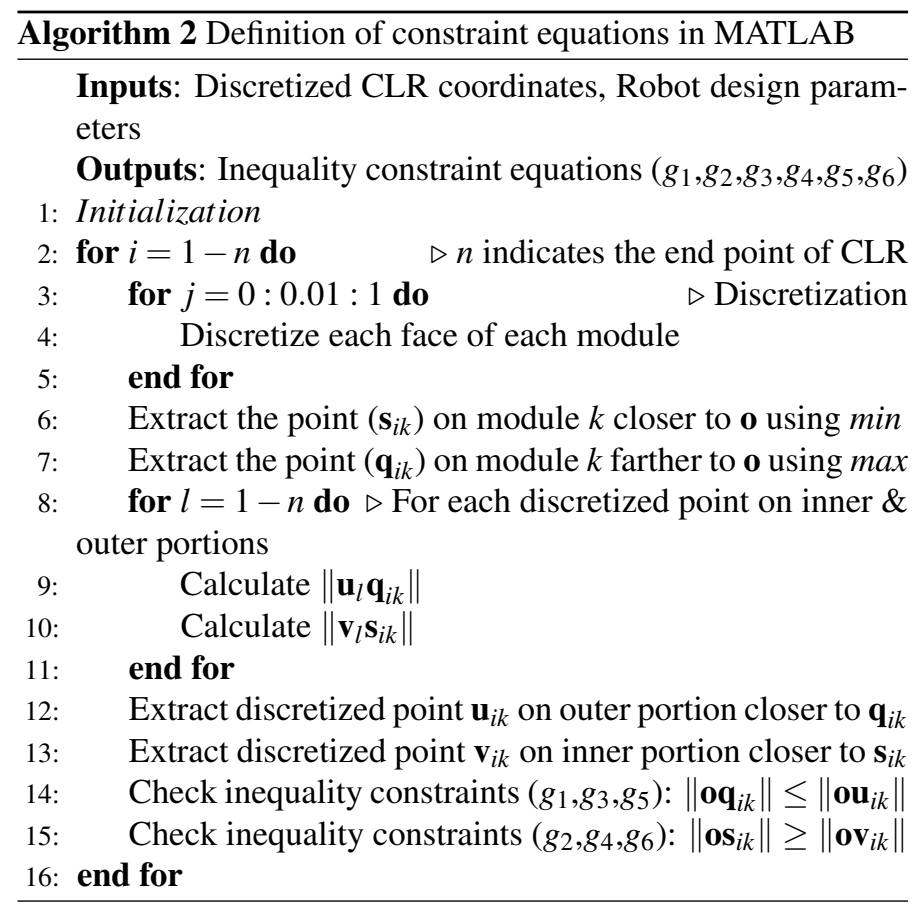

\subsection{Problem statement}

With the objective function and constraint function being defined, the first optimization problem can be stated as

$$
\operatorname{maximize} \sum_{i=1}^{n} f u n 1(\mathbf{x})
$$

subject to constraints: $g_{1}, g_{2}, g_{3}, g_{4}, g_{5}, g_{6}$

$$
\text { where } \mathbf{x}=\left[l_{1}, w_{1}, l_{2}, w_{2}\right]^{T},
$$$$
i=1 . . n \text { indicates the discretized CLR points }
$$

Since identical motor unit is being employed in each module, only 4 design variables are set instead of 12 . The objective and constraint functions are solved using the fmincon function in MATLAB. The design variables are subject to lower and upper bounds to have a closer interpretation of the catalog parts of Maxon. The existing prototype uses a brushless DC-motor of diameter $16 \mathrm{~mm}$ coupled with a spindle drive GP $16 \mathrm{~S}$. The overall length of this assembly is $58 \mathrm{~mm}$ with a screw length of $102 \mathrm{~mm}$. The advantage of using brushless motors is that they offer hall sensors that can be useful in the control phase of the robot to determine pipeline diameters inside unknown/closed environment [16]. The catalog parts of Maxon offers hall sensors for motor diameters starting from $16 \mathrm{~mm}$. The lower $(l b)$ and upper bounds $(u b)$ for the design variables of the optimization problem are set as $l b=[40,8,20,2]$ and $u b=[60,16,102,5]$. The $l b$ of $l_{1}$ and $w_{1}$ are fixed at $40 \mathrm{~mm}$ and $8 \mathrm{~mm}$ as this is the minimum size of motor units offered by Maxon [18]. The $u b$ of $l_{1}$ and $w_{1}$ are taken as $60 \mathrm{~mm}$ and $16 \mathrm{~mm}$ to correlate with the DCMotor unit length and diameter of the existing prototype. The $u b$ of screw length $l_{2}$ is fixed as $102 \mathrm{~mm}$ as this is the maximum size offered by Maxon [18]. Spindle drives of Maxon offer lead screws and ball screws. The existing prototype uses a ball screw type as it has higher load carrying capacities and could be useful when the leg mechanisms are coupled along with the drive. The $u b$ of screw diameter $w_{2}$ is thus set as $5 \mathrm{~mm}$ as per the catalog of Maxon for a $16 \mathrm{~mm}$ diameter spindle drive. The optimization algorithm is executed with a constant value of diameter throughout the section depicted in Fig. 2a. The algorithm will be evaluated one after the other for pipe diameters from 70 to $160 \mathrm{~mm}$. For faster convergence of the problem and also to satisfy bounds at all iterations, the Sequential Quadratic Programming (SQP) algorithm [19] is employed. The tolerance value for the guess variables and the objective function is set at $10^{-9}$.

\subsection{Results and discussions}

As the definition of objective and constraint functions does not have complex parameters, faster convergence is obtained. The dimensions of motor units obtained from the optimization algorithm for the various pipe diameters are provided below in Table 2. From the results of the optimization provided in Table 2, a gradual increase in all design parameters could be observed. The results of $70-80 \mathrm{~mm}$ diameter pipe range provides the least possible sizing. However, it has to be noted that the motor width for these cases is less than $16 \mathrm{~mm}$. This problem can be addressed by using $8 \mathrm{~mm}$ diameter series of Maxon but the feed force and motor efficiency are relatively smaller than the $16 \mathrm{~mm}$ diameter series. Also, the brushless motor series with Hall sensors are available only from $16 \mathrm{~mm}$ diameter motor sizing with Maxon [18]. Thus, the results of 70-80 $\mathrm{mm}$ diameter pipelines will be neglected for further analysis. In the case of a $90 \mathrm{~mm}$ diameter pipeline, better dimensions could be observed but the screw diameter of the spindle drive is found to be $3.9 \mathrm{~mm}$ which is not compatible with the $16 \mathrm{~mm}$ diameter spindle drives of Maxon. Also, the screw length $l_{2}$ might pose a problem when accommodating the slot-follower leg mechanism that will be exploited in the next optimization problem. Thus, the $90 \mathrm{~mm}$ diameter pipeline results will also be not considered for further analysis. Based on the results of Table 2, catalog parts of Maxon are identified for 100 to $160 \mathrm{~mm}$ diameter pipelines and they are provided in Table 3. The EC-motor series 283828 of Maxon [18] is chosen for all the combinations. With this motor, three possi- 
TABLE 2: RESULTS OF OPTIMIZATION PROBLEM-1: SIZING OF MOTOR UNIT

\begin{tabular}{ccccc}
\hline $\begin{array}{c}\text { Pipe diameter } \\
d(\mathrm{~mm})\end{array}$ & $\begin{array}{c}l_{1} \\
(\mathrm{~mm})\end{array}$ & $\begin{array}{c}w_{1} \\
(\mathrm{~mm})\end{array}$ & $\begin{array}{c}l_{2} \\
(\mathrm{~mm})\end{array}$ & $\begin{array}{c}w_{2} \\
(\mathrm{~mm})\end{array}$ \\
\hline 70 & 41.4 & 12.7 & 20 & 2 \\
80 & 47.44 & 14 & 24.7 & 2 \\
90 & 50.95 & 16 & 32 & 3.9 \\
100 & 52.72 & 16 & 45.17 & 5 \\
110 & 55.02 & 16 & 58.25 & 5 \\
120 & 58.21 & 16 & 71.33 & 5 \\
130 & 60 & 16 & 84.4 & 5 \\
140 & 60 & 16 & 86.54 & 5 \\
150 & 60 & 16 & 98.8 & 5 \\
160 & 60 & 16 & 102 & 5 \\
\hline
\end{tabular}

ble spindle drives are identified based on total length, efficiency, speed and feed force factors. The 424731 spindle drive offers the highest velocity. However, the feed force offered by this series is low and might pose a problem during static phases when the leg mechanisms are coupled. The 424744 and 424745 series, on the other hand, have better feed forces. The 424745 series offers a feed force similar to the 424749 series used in the existing prototype. The 424744 series, on the other hand offers, a lesser feed force but this factor will be sufficient to accommodate the leg mechanisms based on experiments conducted on the existing prototype [9]. Also, the velocity offered by 424744 series is much better than the 424745 series which can help address the speed issues of the existing model. Taking into account the length, efficiency and velocity factors, the 283282 EC-motor coupled with 424744 spindle drive is chosen for further analysis. From Table 2, it is interesting to note that with the increase in the diameter of the pipeline, the screw length $l_{2}$ increases. With an $85 \mathrm{~mm}$ screw length, it could be possible to accommodate a leg mechanism that can work between 130-160 mm pipelines. However, with this screw length, it might not be possible to work with 100-120 mm pipeline diameters as there might be collisions when passing through bends. In order to have a working model inside smaller diameter pipelines, the optimization results of $100 \mathrm{~mm}$ are considered for further analysis with which it would be possible to work inside pipe bends having a diameter range between 100 to $120 \mathrm{~mm}$. To ensure a simpler procedure for the control sequence of the prototype, the central module also employs the same motor-spindle combination like the leg modules.

\section{SECOND OPTIMIZATION PROBLEM: DESIGN OF SLOT-FOLLOWER LEG MECHANISM}

With the optimal motor sizing determined from problem-1, the next step is to determine the geometry of leg mechanisms that could be accommodated within the screw length. The ratio of screw length to the pipe diameter has a greater influence on the geometry of leg mechanisms. The geometry of the slot-follower leg mechanism and its assembly on the existing robot is represented below in Fig. 5. By using the inverse kinematic model,

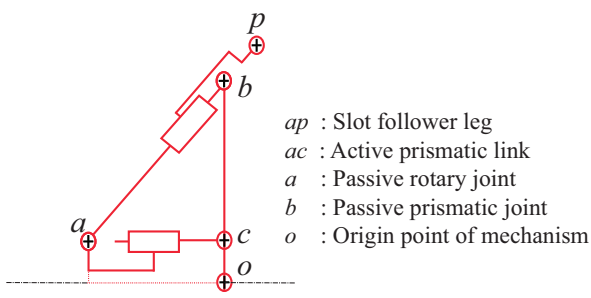

(a)

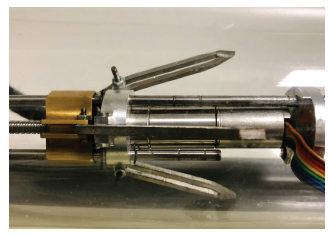

(b)
FIGURE 5: Representation of (a) the geometry of slot-follower leg mechanism ${ }^{1}$ and (b) the assembly of mechanism on the existing prototype $[9,10]$

the dimensions for the leg mechanism were identified in [10] by a multi-objective optimization approach. In this article, a simple mono objective optimization problem is solved where the dimensions of leg mechanisms are determined through a geometrical approach. The maximization of the area occupied by the space of leg mechanism is carried out to determine the maximum leg length that can work inside 100-120 mm diameter pipelines.

\subsection{Modeling of the leg mechanisms and design vari- ables}

The leg mechanism assembly consists of three legs assembled at $120^{\circ}$ with respect to each other [9]. During locomotion, the orientation of leg mechanisms about the axis of robot is unknown. For simplifications, the optimization problem is solved by considering a two-dimensional projection of the geometry of two legs. This geometry of the leg mechanism that will be assembled on the robot is depicted in Fig. 6a. The design parameters for the leg mechanism (Fig. 6c) are the lengths $l_{s 1}, l_{s 2}$, the offsets $o_{1}, o_{2}$, the stroke lengths $\rho_{1}, \rho_{2}$ and the horizontal and vertical space vectors $\Delta \mathbf{x}$ and $\Delta \mathbf{y}[9,10]$. The horizontal and vertical space vector of leg mechanism is given by $\Delta \mathbf{x}=\left[\Delta x_{1}, \Delta x_{2}\right]^{T}$ and

\footnotetext{
${ }^{1}$ Simulation of the leg mechanism and robot in CATIA https: / / www - youtube. com/watch?v=7 z 6-by $83 \mathrm{mtw}$
} 
TABLE 3: MAXON CATALOG PARTS IDENTIFICATION WITH RESPECT TO RESULTS OF OPTIMIZATION FOR 100 TO $160 \mathrm{~mm}$ DIAMETER PIPES

\begin{tabular}{cccccc}
\hline Type & Series & Gear ratio & $\begin{array}{c}\text { Feed force } \\
(\mathrm{N})\end{array}$ & $\begin{array}{c}\text { Efficiency } \\
(\%)\end{array}$ & $\begin{array}{c}\text { Length (Motor+Spindle) } \\
(\mathrm{mm})\end{array}$ \\
\hline Motor & 283828 & $5.4: 1$ & 189 & 87 & 46.4 \\
Spindle & 424731 & & & & \\
Motor & 283828 & $29: 1$ & 331 & 79 & 51.5 \\
Spindle & 424744 & & & & \\
Motor & 283828 & $104: 1$ & 403 & 71 & 55.1 \\
Spindle & 424745 & & & & \\
\hline
\end{tabular}

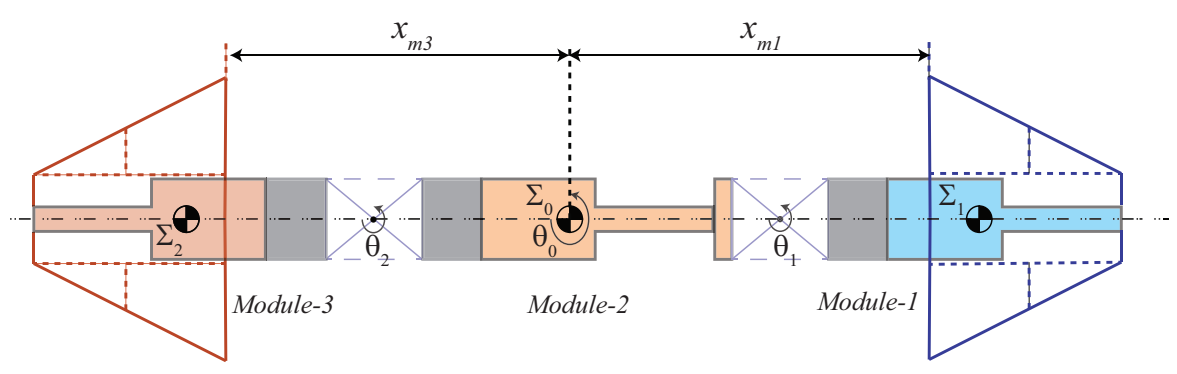

(a)

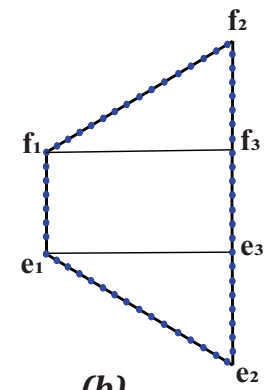

(b)
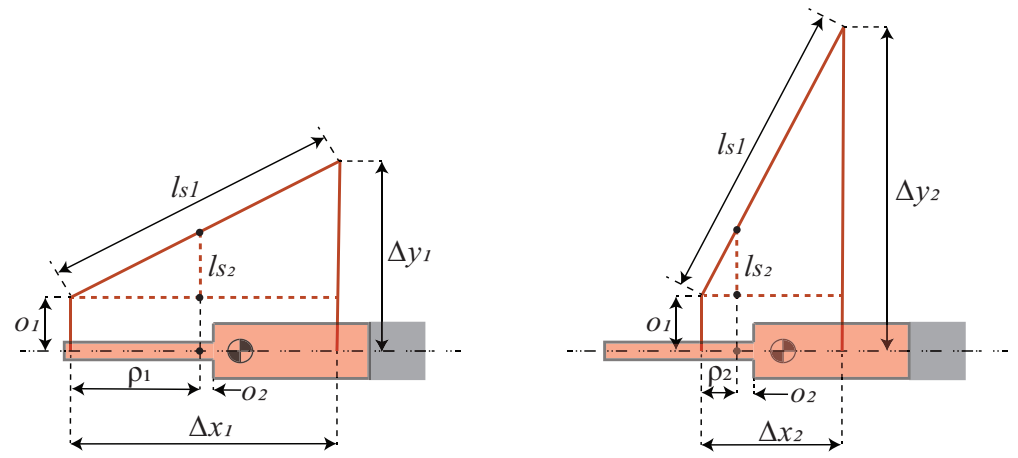

(c)

FIGURE 6: Representation of (a) the slot-follower leg mechanism on the robot, (b) the discretization of leg mechanism and (c) the design parameters defining the geometry of leg mechanism during declamping and clamping phases

$\Delta \mathbf{y}=\left[\Delta y_{1}, \Delta y_{2}\right]^{T}$. During locomotion of robot inside the pipeline, at least one set of leg mechanisms establish contact with pipeline walls. However, this sequence will be time-consuming to construct in MATLAB. In order to have faster convergence and a simpler model, it is assumed that at all positions of CLR both the set of leg mechanisms remain in the declamped phase during simulation. The design variables for the optimization problem is given by $\left[\Delta x_{1}, \Delta y_{1}, l_{s 2}\right]$. The offsets $o_{1}$ and $o_{2}$ are retained as $11 \mathrm{~mm}$ and $7 \mathrm{~mm}$, based on the existing prototype as these correspond to offsets caused by the EC-Motor unit $\left(o_{1}\right)$ and spacers $\left(o_{2}\right)$ used for assembly of leg mechanisms. The parameter $l_{s 2}$ represents the vertical distance of the hinge point of leg mecha- 
nism. This parameter plays an essential role to determine the leg length $l_{s 1}$ for a given diameter range of pipeline. The parameter $\Delta x_{1}$ is referenced from the frame $\sum_{0}$ using $x_{m 3}$ for Module- 3 and $x_{m 1}$ for Module-1 as depicted in Fig. 6a.

\subsection{Objective function}

Since no leg masses are attached to the central motor, this module will not be taken into account for the second optimization problem. The objective function aims to maximize the space occupied by the leg mechanisms. This space can be split into triangular and rectangular areas as represented in Fig. $6 \mathrm{~b}$. The area of the leg mechanisms used in the robot at a given discretized CLR point can be calculated by

fun $2=\sum_{k=1}^{2}\left(\frac{\left\|\mathbf{e}_{k 1} \mathbf{e}_{k 3}\right\|\left\|\mathbf{e}_{k 3} \mathbf{e}_{k 2}\right\|}{2}+\frac{\left\|\mathbf{f}_{k 1} \mathbf{f}_{k 3}\right\|\left\|\mathbf{f}_{k 3} \mathbf{f}_{k 2}\right\|}{2}+\left\|\mathbf{e}_{k 1} \mathbf{e}_{k 3}\right\|\left\|\mathbf{e}_{k 1} \mathbf{f}_{k 1}\right\|\right)$

where $k=1,2$ indicates Module- 1 and Module- 3

Similar to Eqn. (4), the global sum of fun2 for Eqn. (7) is estimated which will be maximized.

\subsection{Constraint function}

At all positions of CLR, the collision of the geometry of leg mechanisms is verified against the pipeline walls. Inequality constraints from Algorithm 2 are carried over to this optimization problem which will ensure that the discretized geometry of the leg mechanism avoids collisions with inner and outer portions of the pipe. However, the inequality constraints are reduced from 6 to 4 for this problem as the central module is not taken into account. The inequality constraint equations are similar to Eqn. (5) \& Eqn. (6) but instead, they apply only to the front and rear modules. Two additional equality constraints will be defined for this optimization problem. As the simulation is performed for fully declamped phases, the length of leg mechanism $l_{s 1}$ can be calculated from Fig. 6b using Pythagoras theorem and it is given by

$$
l_{s 1}(d)=\sqrt{\left\|\mathbf{f}_{k 1} \mathbf{f}_{k 3}\right\|+\left\|\mathbf{f}_{k 2} \mathbf{f}_{k 3}\right\|}
$$

where $i=1,2$ indicates Module- 1 and Module-3

In Eqn. (8), $l_{s 1}(d)$ indicates the leg length during declamped phase. During the clamped phase, the length $l_{s 1}$ can be calculated with the help of $\Delta x_{2}, \Delta y_{2}$ and $o_{1}$ by the Pythagoras theorem. Also during the declamped phase, the parameter $l_{s 2}$ can be estimated using similar triangles. The equations are given by

$$
\begin{aligned}
l_{s 1}(c) & =\sqrt{\Delta x_{2}^{2}+\left(\Delta y_{2}-o_{1}\right)^{2}} \\
\frac{l_{s 2}}{\rho_{1}} & =\frac{\Delta y_{1}-o_{1}}{\Delta x_{1}}
\end{aligned}
$$

In Eqn. (9), $l_{s 1}(c)$ is the length of the leg mechanism during clamped phase. The stroke lengths $\rho_{1}$ and $\rho_{2}$ are set as $32 \mathrm{~mm}$ and $7 \mathrm{~mm}$ with reference to the $45 \mathrm{~mm}$ screw length of motor unit for $100 \mathrm{~mm}$ diameter pipe (Table 2). The reduction in stroke length is caused by flanges and fasteners that will be used for the robot assembly. The two equality constraints for the second optimization problem are thus given by

$$
\begin{aligned}
h_{1}: \rho_{1} & =32 \\
h_{2}: l_{s 1}(d) & =l_{s 1}(c)
\end{aligned}
$$

\subsection{Problem statement}

For the second optimization problem, the diameter $d$ of the pipeline is fixed as $100 \mathrm{~mm}$. The optimization problem to estimate the size of the leg mechanisms can be defined by

$$
\begin{gathered}
\text { maximize } \sum_{i=1}^{n} f u n 2(\mathbf{z}) \\
\text { subject to constraints: } g_{1}, g_{2}, g_{3}, g_{4}, h_{1}, h_{2} \\
\text { where } \mathbf{z}=\left[\Delta x_{1}, \Delta y_{1}, l_{s 2}\right]^{T}, \\
i=1 . . n \text { indicates the discretized CLR positions }
\end{gathered}
$$

The lower and upper bounds of the design parameters for the simulation are given by $l b=[40,20,5], u b=[65,40,12]$. In Eqn. (9), the value of $\Delta y_{2}$ is fixed as $60 \%$ of the pipe diameter taken for simulation. A value higher than $60 \%$ leads to convergence issues for a screw length of $45 \mathrm{~mm}$. With the limit of $\rho_{2}$ set to $7 \mathrm{~mm}$, the value of $\Delta x_{2}$ can be calculated using similar triangles. Using the fmincon function with the SQP algorithm, the optimization problem is solved to determine the geometry of the leg mechanism.

\subsection{Results and discussions}

The dimensions of the slot-follower leg mechanism after optimization are provided in Table 4. Compared to the existing prototype, the parameters $l_{s 1}$ and $l_{s 2}$ are increased from $57 \mathrm{~mm}$ and $7 \mathrm{~mm}$ to $62.7 \mathrm{~mm}$ and $9.7 \mathrm{~mm}$ respectively. In the event of a straight pipeline with no bends, the slot-follower leg mechanism is capable of adjusting its size between $58.6 \mathrm{~mm}\left(2 \Delta y_{1}\right)$ to $124 \mathrm{~mm}\left(2 \Delta y_{2}\right)$ diameter range. When the robot is moving inside a pipeline with bends, the modules are capable of passing 


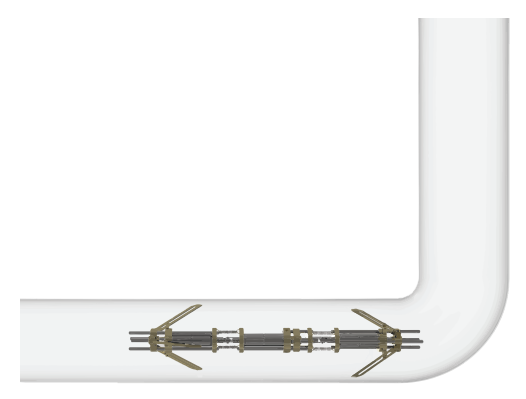

(a)

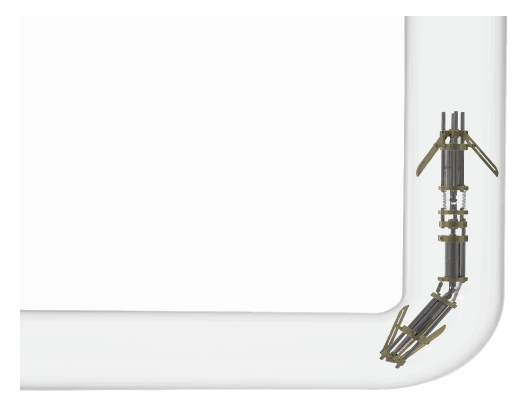

(d)

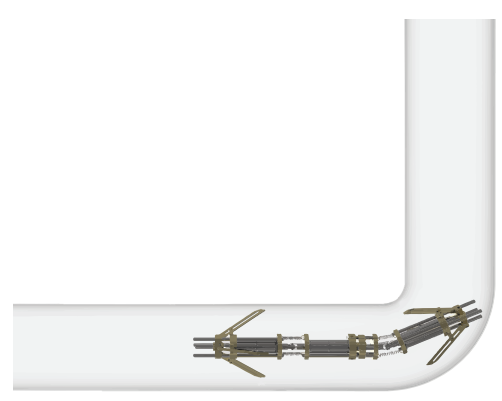

(b)

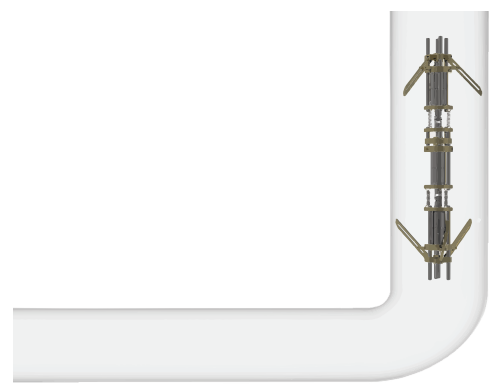

(e)

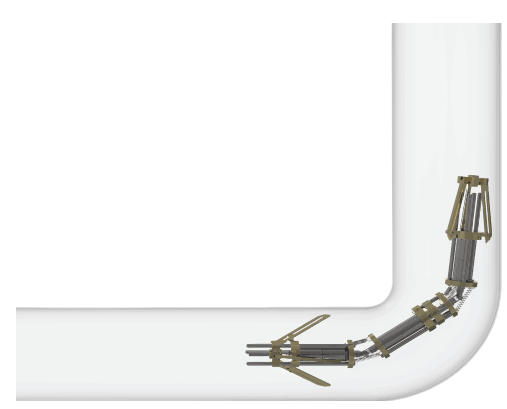

(c)

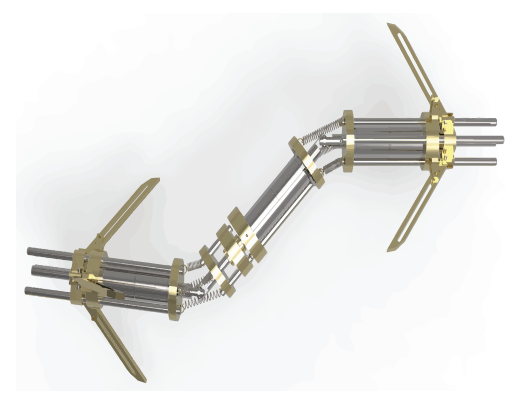

(f)

FIGURE 7: Locomotion sequence of the optimized bio-inspired robot inside a $100 \mathrm{~mm}$ diameter pipe from (a) to (e) and correlation of the robot to an "Elephant trunk" (f)

TABLE 4: RESULTS OF OPTIMIZATION PROBLEM-2: SLOT-FOLLOWER DIMENSIONS

\begin{tabular}{cc}
\hline Parameters & Dimensions $(\mathrm{mm})$ \\
\hline$\Delta x_{1}$ & 60 \\
$\Delta y_{1}$ & 29.3 \\
$\Delta y_{2}$ & 62 \\
$l_{s 1}$ & 62.7 \\
$l_{s 2}$ & 9.7 \\
\hline
\end{tabular}

through such bends, provided the diameter of pipelines is strictly between 100 to $120 \mathrm{~mm}$. By assembling the tensegrity mechanism, the EC-motor with spindle drive unit identified from optimization problem-1, the optimized slot-follower leg mechanism and standard fasteners such as circlips, bolts, etc., the entire robot can be realized using CATIA software. The locomotion of the robot inside a $100 \mathrm{~mm}$ diameter is rendered using CATIA and the sequence is represented in Fig. 7a to Fig. 7e. The reconfigurable robot resembles an "Elephant trunk" and it is demonstrated in Fig. 7f.

\section{CONCLUSIONS}

In this article, an optimization approach was employed to determine the sizing of the robot and its associated leg mechanism to pass through pipe bends. Two problems were defined and solved in this article which employed the deterministic optimization approach in MATLAB. The first problem dealt with the estimation of sizes of individual motor modules without the presence of leg mechanisms inside a test bench with different diameters. From the results of optimization, the dimensions for $100 \mathrm{~mm}$ diameter pipe were chosen to have a nominal screw length that can accommodate the slot-follower leg mechanism for the second problem. The catalog parts of Maxon were identified based on the results of the first optimization problem. Also, a motorspindle combination with Hall sensor unit and better velocity ratio for faster displacements was identified for the reconfigurable robot. Followed by that, in the second problem the sizing of the slot-follower leg mechanism was determined by a geometric approach that did not require the kinematic models. The dimensions of the slot-follower leg mechanism were found to be in- 
creased when compared to the existing prototype. However, with the modified architecture, the robot is capable of working inside 100-120 mm diameter pipeline range that can have straight sections and bends at $90^{\circ}$. The presence of leg mechanisms and tensegrity mechanism permits to have a reconfigurable "Elephant trunk" model that allows the robot to pass through pipe bends.

In future works, the optimization algorithm can be modified to accommodate a relation between screw length and pipeline diameter thereby reducing user efforts to fix the pipeline diameters for each computation. This modification might probably lead to the definition of another objective and evolutionary algorithms will be implemented to solve this problem. With the $3 \mathrm{D}$ model being assembled using catalog parts, the static force model developed in [9] can be extended to the reconfigurable robot. This model can help in determining the forces on the tensegrity mechanisms as well as the maximum clamping forces which can be useful for performing experiments. A simulation of the robot will be performed using ADAMS software to validate the locomotion procedure followed by which prototyping of the reconfigurable robot will be done. For the real-time application, suitable control units and a camera will be accommodated on the robot to perform an inspection of pipelines. Based on simulation trails, an investigation will also be done to replace the central module with a spindle drive having higher velocity for accomplishing faster displacements.

\section{REFERENCES}

[1] Choi, H. R., and Roh, S.-g., 2007. "In-pipe robot with active steering capability for moving inside of pipelines". In Bioinspiration and Robotics Walking and Climbing Robots. IntechOpen.

[2] Okamoto Jr, J., Adamowski, J. C., Tsuzuki, M. S., Buiochi, F., and Camerini, C. S., 1999. "Autonomous system for oil pipelines inspection”. Mechatronics, 9(7), pp. 731-743.

[3] Okada, T., and Kanade, T., 1987. "A three-wheeled selfadjusting vehicle in a pipe, ferret-1". The International journal of robotics research, 6(4), pp. 60-75.

[4] Roman, H. T., Pellegrino, B., and Sigrist, W., 1993. "Pipe crawling inspection robots: an overview". IEEE transactions on energy conversion, 8(3), pp. 576-583.

[5] Ryew, S., Baik, S., Ryu, S., Jung, K. M., Roh, S., and Choi, H. R., 2000. "In-pipe inspection robot system with active steering mechanism". In Proceedings. 2000 IEEE/RSJ International Conference on Intelligent Robots and Systems (IROS 2000)(Cat. No. 00CH37113), Vol. 3, IEEE, pp. 1652-1657.

[6] Neubauer, W., 1994. "A spider-like robot that climbs vertically in ducts or pipes". In Proceedings of IEEE/RSJ International Conference on Intelligent Robots and Systems (IROS'94), Vol. 2, IEEE, pp. 1178-1185.

[7] Fukuda, T., Hosokai, H., and Uemura, M., 1989. "Rub- ber gas actuator driven by hydrogen storage alloy for inpipe inspection mobile robot with flexible structure". In Proceedings, 1989 international conference on robotics and automation, IEEE, pp. 1847-1852.

[8] Horodinca, M., Doroftei, I., Mignon, E., and Preumont, A., 2002. "A simple architecture for in-pipe inspection robots". In Proc. Int. Colloq. Mobile, Autonomous Systems, pp. 6164.

[9] Venkateswaran, S., Chablat, D., and Boyer, F., 2019. "Numerical and experimental validation of the prototype of a bio-inspired piping inspection robot'. Robotics, 8(2), p. 32 .

[10] Henry, R., Chablat, D., Porez, M., Boyer, F., and Kanaan, D., 2014. "Multi-objective design optimization of the leg mechanism for a piping inspection robot". In Proceedings of the ASME 2014 IDETC-CIE, Vol. 5A: 38th Mechanisms and Robotics Conference. V05AT08A001.

[11] Chablat, D., Venkateswaran, S., and Boyer, F., 2019. "Dynamic model of a bio-inspired robot for piping inspection". In ROMANSY 22-Robot Design, Dynamics and Control. Springer, pp. 42-51.

[12] Venkateswaran, S., and Chablat, D., 2019. "A new inspection robot for pipelines with bends and junctions". In IFToMM World Congress on Mechanism and Machine Science, Springer, pp. 33-42.

[13] Guessasma, S., and Bassir, H., 2009. "Comparing heuristic and deterministic approaches to optimise mechanical parameters of biopolymer composite materials". Mechanics of Advanced Materials and Structures, 16(4), pp. 293-299.

[14] Rao, R. V., and Waghmare, G., 2017. "A new optimization algorithm for solving complex constrained design optimization problems". Engineering Optimization, 49(1), pp. 60-83.

[15] Venkateswaran, S., Furet, M., Chablat, D., and Wenger, P., 2019. "Design and analysis of a tensegrity mechanism for a bio-inspired robot". In Proceedings of the ASME 2019 IDETC-CIE, Vol. 5A: 43rd Mechanisms and Robotics Conference. V05AT07A026.

[16] Venkateswaran, S., Chablat, D., and Ramachandran, R., 2019. "Prototyping a piping inspection robot using a beaglebone black board”. In 24éme Congrès Français de Mécanique, Brest.

[17] Li, H., Yang, H., Zhan, M., and Gu, R., 2007. "The interactive effects of wrinkling and other defects in thin-walled tube nc bending process". Journal of Materials Processing Technology, 187, pp. 502-507.

[18] Maxon Motors, Program 2017/18. High precision Drives and Systems. http://epaper.maxonmotor.com/. Accessed: 2019-12-15.

[19] Chosing the algorithm- MATLAB \& Simulink- Mathworks France. https://fr.mathworks.com/help/ optim/ug/choosing-the-algorithm.html. Accessed: 2020-02-02. 